\title{
The evidence base for rectal prolapse surgery: is resection rectopexy worth the risk?
}

\author{
S. Brown
}

Received: 31 July 2013/Accepted: 15 September 2013/Published online: 1 October 2013

(C) Springer-Verlag Italia 2013

The optimal operation for rectal prolapse is unclear. Various options exist. Broadly, one can carry out the operation via a perineal or abdominal approach. If perineal, should it be a Delormes or rectosigmoidectomy combined with a levatorplasty or not? If abdominal, should it be open or laparoscopic and should the rectum be simply mobilised or fixed? If fixed, what with and where from and to? Should the lateral ligaments be divided or preserved and should the redundant sigmoid be resected?

With such a huge variation in techniques, what is the evidence that will tell us what the best approach for prolapse surgery is? Unfortunately, the literature is full of poor quality data mainly from case series and expert opinion. So the first step must be to concentrate on only the quality comparative data in the form of randomised controlled trials. Can this data tell us anything?

The next step is to decide what outcome to assess. The obvious outcome is recurrence. When considering the two broad approaches, there is a perception that an abdominal procedure will result in a lower incidence of recurrence. Indeed, the recurrence rate for any perineal procedure may be greater than $40 \%$ if the patient is followed up for long enough [1]. Is there good quality comparative data to back up this perception that the abdominal procedure is more robust? Two randomised trials have taken on this comparison $[1,2]$. Both have inadequate numbers of patients to

Comment on El Muhtaseb et al.: Colonic transit before and after resection rectopexy for full-thickness rectal prolapse (doi:10.1007/s10151-013-1053-4).

\section{S. Brown $(\square)$}

Department of Surgery, Sheffield Teaching Hospitals, Northern General Hospital, Herried Road, Sheffield S7, South Yorkshire S5 7AU, UK

e-mail: Steven.Brown@sth.nhs.uk reach any meaningful conclusion, but for the numbers that have been examined no difference in recurrence was seen for either approach. The most recent trial failed to recruit adequate numbers despite the trial being multicentre, indeed multinational, and running for many years.

Herein lies the problem with such trials. Surgeons are inclined to individualise when it comes to approach, with the perineal procedure, perhaps done under regional anaesthetic, reserved for elderly frail patients, and the abdominal approach indicated for younger patients who can tolerate a more invasive procedure and have potentially many more years of life. Equipoise does not therefore exist, and any trial becomes very difficult to recruit to. But perhaps it is time for a readjustment of this surgical dogma; the advent of laparoscopic techniques means that elderly patients can undergo abdominal procedures without an increase in complications [3].

If we assume the decision has been made to carry out an abdominal approach, is there any evidence to suggest how to carry out the operation? In terms of recurrence there probably is not. However, one large reasonably welldesigned trial has shown that the rectum cannot simply bemobilised; it has to be fixed to reduce recurrence [4]. However, recurrence is not the only important outcome. For instance, in terms of recovery, it is clear that a laparoscopic approach results in shorter postoperative stay and actually reduces overall costs [5].

Constipation is another important outcome, and the common perception is that abdominal rectopexy alone will increase the incidence of constipation in many patients, justifying the need for resection to be combined with the rectopexy [6]. Is this true? Is the added possibility of anastomotic breakdown, even if rare, really worth the risk? The study by El Muhtaseb et al. [7] would suggest it is not, with resection rectopexy failing to correct abnormal transit 
despite resection of a significant length of bowel (at least $60 \mathrm{~cm}$ ). The authors suggest most patients have a pancolonic motility disorder that is not corrected with segmental colonic resection. However, this result is at odds with the existing quality comparative data which suggests that resection rectopexy does reduce the potential for constipation postoperatively [5].

How do you explain this discrepancy? The key may be in the technique of rectal mobilisation. Division of all lateral attachments to the rectum was undertaken routinely in this study. As the authors point out, this has the potential to denervate the rectum by damaging the parasympathetic supply via the inferior hypogastric nerve [8]. Certainly, the comparative data that exist suggest division, although improving recurrence rates, probably results in a higher incidence of postoperative constipation [5]. As El Muhtasab et al. did not detail segmental transit marker times, it is possible that in some patients, the markers were grouped in the distal bowel due to rectal inertia.

So what can we tell from this study and the limited quality comparative data on rectal prolapse surgery? Essentially, there is a need for more well-designed trials. An abdominal procedure should undoubtedly be done laparoscopically, and if we as surgeons become confident that this laparoscopic approach can be used safely in the elderly, it is perhaps time for another attempt at an abdominal/perineal trial. Such a trial is being developed in Germany (The DeloRes trial) [9]. It is interesting that the investigators of this trial feel that the best abdominal comparator is laparoscopic resection rectopexy suggesting this procedure remains popular in Europe as well as the USA [10]. If an abdominal procedure is indicated, whether combined with resection or not, we need to know how to fix the rectum. A proposed Danish trial (the Laparoscopic Rectopexy trial) may well answer this question [11]. Both this trial and a proposed UK trial (the Delivar trial) utilise another increasingly popular treatment for prolapse surgery, laparoscopic ventral rectopexy, as one comparator. This operation, which is alluded to by El Muhtaseb et al. in their discussion, appears to meet all the criteria for the perfect prolapse operation; it is minimally invasive, it does not involve division of the lateral ligaments and therefore should not induce constipation, and it appears to be associated with a low recurrence rate [12]. The trouble is it has never been tested in any high-quality comparative trial. So these two trials should be supported strongly, and the results should be awaited with eager anticipation. Perhaps there is room for another trial comparing laparoscopic ventral rectopexy with resection rectopexy that could finally tell us whether resection really is worth the risk. In the meantime, let us hope the ogre of mesh erosion does not rear its ugly head.

Conflict of interest None.

\section{References}

1. Senapati A, Gray RG, Middleton LJ et al (2013) PROSPER: a randomised comparison of surgical treatments for rectal prolapse. Colorectal Dis 15:858-868

2. Deen KI, Grant E, Billingham C, Keighley MRB (1994) Abdominal resection rectopexy with pelvic floor repair versus perianal rectosigmoidectomy and pelvic floor repair for fullthickness rectal prolapse. Br J Surg 81:302-304

3. Wijffels N, Cunnigham C, Dixon A, Greenslade G, Lindsay I (2011) Laparoscopic anterior rectopexy for external rectal prolapse is safe and effective in the elderly. Does this make perineal approach obsolete? Colorectal Dis 13:561-566

4. Karas JR, Uranues S, Altomare DF et al (2011) No rectopexy versus rectopexy following rectal mobilisation for full-thickness rectal prolapse: a randomised controlled trial. Dis Colon Rectum 54:29-34

5. Tou S, Brown SR, Malik AI, Nelson RL (2008) Surgery for complete rectal prolapse in adults. Cochrane Database Syst Rev 8:CD001758

6. Laubert T, Kleemann M, Roblick UJ et al (2013) Obstructive defecation syndrome: 19 years of experience with resection rectopexy. Tech Coloproctol 17:307-314

7. El Muhtaseb MS, Bartolo DC, Zayiae D, Salem T (2013) Colonic transit before and after resection rectopexy for full-thickness rectal prolapse. Tech Coloproctol. doi:10.1007/s10151-013-1053-4

8. Varma JS (1992) Autonomic influences on colorectal motility and pelvic surgery. World J Surg 16:811-919

9. Rothenhoefer S, Herrle F, Herold A et al (2012) DeloRes trial: study protocol for a randomized controlled trial comparing two standardized approaches in rectal prolapse-Delorme's procedure versus resection rectopexy. Trials 13:155

10. Magruder JT, Efron JE, Wick EC, Gearhart SL (2013) Laparoscopic rectopexy for rectal prolapse to reduce surgical site infections and length of stay. World J Surg 37:1110-1114

11. Iverson LH, Laurberg S The laparoscopic rectopexy trial. http:// clinicaltrials.gov/show/NCT00946205

12. Samaranayake CB, Luo C, Plank AW, Merrie AE, Plank LD, Bisset IP (2010) Systematic review on ventral rectopexy for rectal prolapse and intussusception. Colorectal Dis 12:504-514 